\title{
Virtual care is here to stay, but major challenges remain
}

\author{
n Cite as: CMAJ 2020 July 27;192:E868-9. doi: 10.1503/cmaj.1095884
}

Posted on cmajnews.com on July 9, 2020

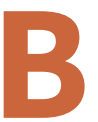
efore the pandemic, Vancouver resident Sarah Hornstein didn't like the idea of virtual health care. Newly diagnosed with asthma shortly before the shutdown, she worried that virtual care would be "more about efficiency" than quality. "It's hard enough to get a doctor's time," Hornstein says.

But after several virtual care visits, Hornstein says she was "surprised by how well it's working." She appreciated that she could go about her day until her doctor called, instead of waiting around if other appointments ran late. And the providers she spoke with answered all her questions, with one appointment lasting half an hour. Although Hornstein says she would still opt for in-person visits "most of the time," she now prefers phone appointments for exchanging information.

During the pandemic, virtual care went from being extremely rare to "the default," largely by necessity, says Dr. Ewan Affleck, cochair of the Canadian Medical Association (CMA)'s virtual care task force. According to a Canada Health Infoway survey, more than half of Canadians said their most recent health encounters in April, May and June this year were virtual.

The introduction of fee codes allowing doctors to bill provincial health plans for virtual care removed the main financial barrier to broader uptake by physicians and patients. But other longstanding barriers to virtual care remain.

"The horse has left the barn..." Health authorities have mostly left doctors to figure out which technologies to use, when to use them, and how to work around licensing and jurisdictional issues.

"The horse has left the barn and we're scrambling to put on the saddle," says
Dr. Gigi Osler, past president of the CMA and an otolaryngology-head and neck surgeon in Winnipeg. "There is very little governance in this area."

Some provinces like Nova Scotia stipulated specific platforms to use, while others like Ontario left the choice to physicians. The Ontario Telemedicine Network (OTN) recommended technical and security standards as a guide. But in the rush to scale up virtual care during the pandemic, "people had to just get something," says OTN CEO Dr. Ed Brown. "I hope they're reasonably secure."

Meanwhile, medical licensing rules continue to pose a challenge, especially in northern communities, where patients often must access specialist care in other jurisdictions. Some provincial medical regulators relaxed restrictions on virtual care during the pandemic, but licensing requirements still vary across the country. And according to Affleck, who is based in Yellowknife, "non-aligned policy environments" can prevent providers from sharing patient information across jurisdictional boundaries.

\section{New clinical challenges}

Physicians are also struggling with making clinical decisions when virtual care is the default. Dr. Michelle Cohen, a

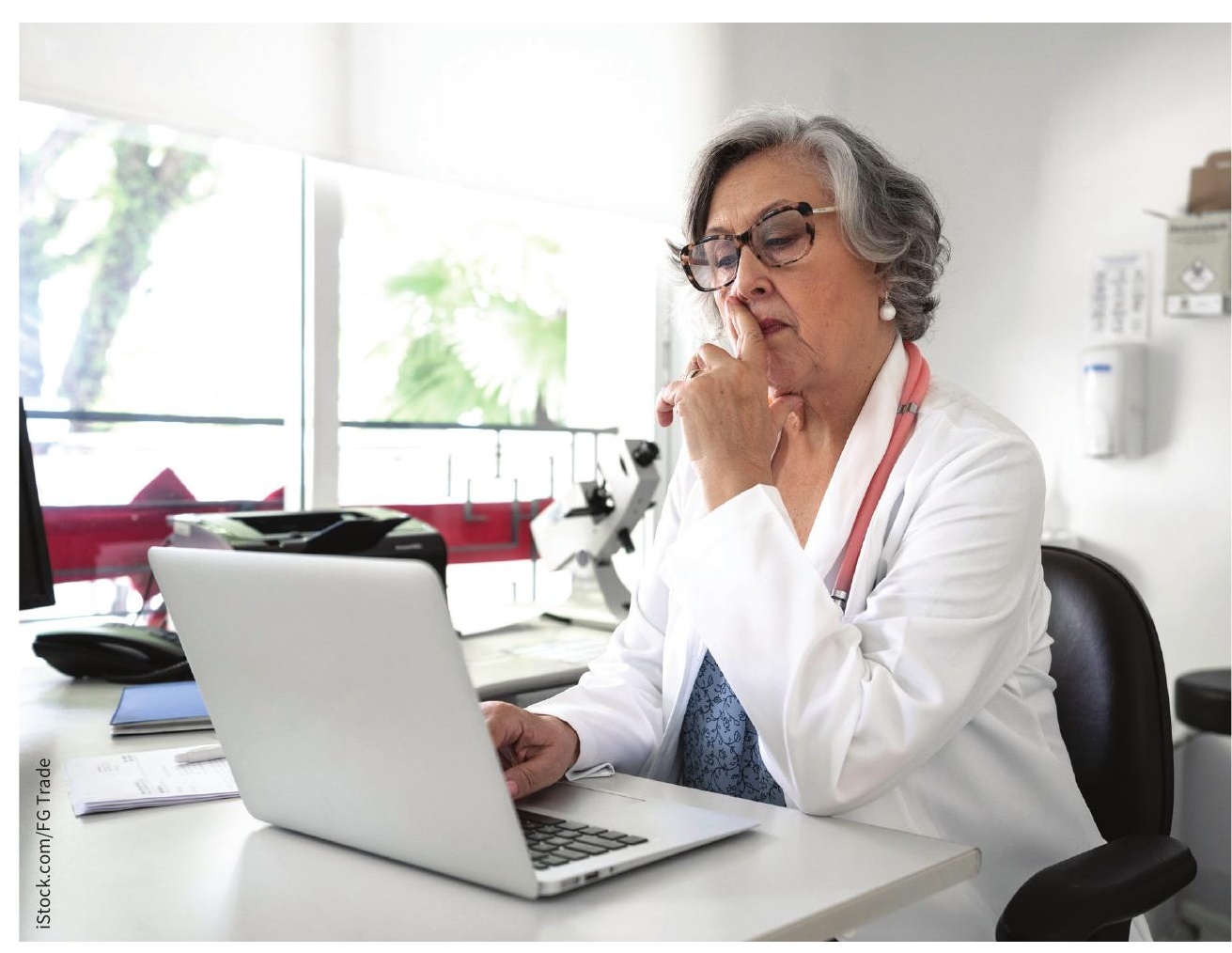

Health authorities have mostly left physicians to figure out how to provide virtual care during the pandemic. 
family and emergency doctor in Brighton, Ont., says she has found it difficult to weigh the risk of missing something important in a virtual visit against the risk of seeing patients in-person and potentially exposing them to coronavirus disease 2019 (COVID-19).

Cohen has tried to talk patients through physical exam manoeuvres, like the ones she would use to diagnose a hernia, but says those attempts have "utterly failed." In many cases, she sets a reminder to check in with patients a week or two later to see if their symptoms have changed. Often, she ends up seeing those patients in person.

Dr. Ritika Goel, a family doctor in Toronto, says that social and economic factors can also make it difficult to know whether to see a patient virtually. Marginalized patients may not be able to advocate for in-person appointments when they need them, and lower levels of health literacy and mental health issues can impede phone conversations. Many of Goel's patients haven't been able to make phone appointments because they may not have access to a charged phone.

While the pandemic has complicated decisions about when to use virtual care, Brown says that physicians can generally rely on the same clinical judgement they use when deciding whether to schedule an appointment with a patient or send them to the emergency department. However, he notes that there are some additional factors to consider. "What's their cognitive capacity? How much does a virtual appointment help them? Are they going to be able to avoid time off work? Are there language barriers? Are there mobility issues?"

As the pandemic wears on, weighing virtual appointments versus in-person visits is "increasingly becoming intuitive for providers," says Brown. But in the long term, doctors will likely need additional training in virtual care.

According to Affleck, "there's not a systematic approach to the integration of virtual care into the curriculum of most medical schools in Canada." And medical licensing bodies don't evaluate physician's clinical judgement regarding virtual care.

Despite these challenges, many physicians and patients are embracing virtual care. Cohen predicts that most follow-up appointments "are probably going to end up being phone calls in the future," even after physical distancing is no longer necessary.

Osler says that feedback from her patients on the switch to virtual care has been "overwhelmingly positive." She has found that phone appointments work well for history taking and following up on test results, rather than scheduling inperson appointments that may not be optimally timed. "For my patients, the virtual care often feels more complete to them," Osler says.

Wendy Glauser, Toronto, Ont. 